\title{
Preocupación por el cambio climático, condiciones económicas individuales y priorización del medioambiente en América Latina
}

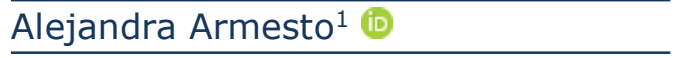

Los ciudadanos de América Latina se preocupan por las consecuencias del cambio climático más que los de cualquier otra región del mundo. Sin embargo, esta preocupación no siempre conduce a la priorización del medioambiente sobre el crecimiento económico. Este artículo argumenta que los constreñimientos económicos de los individuos condicionan la relación entre sus creencias acerca de la gravedad de las consecuencias del cambio climático y sus preferencias frente al dilema entre priorizar el medioambiente o el crecimiento económico. El análisis de encuestas del Barómetro de las Américas (Lapop) de 2016 en 18 países de América Latina, con modelos jerárquicos lineales y ecuaciones estructurales generalizadas, muestra que las creencias acerca de la seriedad de las consecuencias del cambio climático tienen un efecto positivo y significativo sobre la priorización del medioambiente entre los individuos con una riqueza patrimonial por encima de la media y un efecto negativo entre los individuos con riqueza patrimonial por debajo de la media. Este artículo hace dos contribuciones. Primero, el estudio analiza el efecto condicionado de la preocupación por el cambio climático sobre la priorización del medioambiente dependiendo de los constreñimientos económicos individuales. En segundo lugar, aporta a la investigación acerca de las actitudes medioambientales en América Latina.

Palabras clave: actitudes ambientales; preocupación por el cambio climático; priorización del medioambiente; condiciones económicas; modelos jerárquicos lineales; América Latina

\section{Introducción}

En 2015, la Encuesta Global del Pew² Center encontró que, en América Latina, más de dos tercios de su población consideraba que las consecuencias del cambio climático serían muy serias. Aunque alrededor del mundo la mayoría de la gente cree que el cambio climático global es un problema apremiante, el nivel de preocupación de los latinoamericanos supera el de cualquier otra región del mundo (Dayrell, 2019; Dayrell y

\footnotetext{
${ }^{1}$ Facultad Latinoamericana de Ciencias Sociales. Mexico City, Mexico.

E-mail: <alejandra.armesto@flacso.edu.mx>.

2 Disponible en: <https://www.pewresearch.org/global/2015/06/23/spring-2015-survey/>. Accesado el: fev. 2021.
} 
PREOCUPACIÓN POR EL CAMBIO CLIMÁTICO, CONDICIONES ECONÓMICAS INDIVIDUALES Y PRIORIZACIÓN DEL MEDIOAMBIENTE..

Urry, 2015; Stokes, Wike y Carle, 2015). Sin embargo, esta percepción subjetiva de la gravedad del cambio climático no se asocia inequívocamente a la priorización del medioambiente sobre el crecimiento económico. ¿Bajo qué condiciones la preocupación por las consecuencias del cambio climático conduce a priorizar el medioambiente?

El artículo responde a la pregunta planteada atendiendo a los constreñimientos económicos que moderan la relación entre la preocupación por el calentamiento global y las preferencias con respecto al dilema entre priorizar el medioambiente o el crecimiento económico (Franzen, 2003). La prosperidad económica ofrece niveles de bienestar que permiten a los individuos elegir y, dada su percepción de la seriedad de las consecuencias del cambio climático, decidir si prioriza el medioambiente o el crecimiento económico. Por el contrario, las condiciones económicas adversas restringen las oportunidades de los individuos para decidir. Quienes no tienen niveles básicos de bienestar, aun cuando exhiben preocupación por las consecuencias del calentamiento global, pueden encontrar muy costoso renunciar al crecimiento económico. Dados estos constreñimientos, hipotetizo que el nivel de preocupación por el cambio climático tendrá efectos sobre priorización del medioambiente a expensas del crecimiento, dependiendo de la situación económica individual.

El artículo prueba este argumento analizando datos de las encuestas del año 2016 del Proyecto de Opinión Pública de América Latina (Lapop) en dieciocho países latinoamericanos ${ }^{3}$. América Latina ofrece un buen caso para estudiar las actitudes con respecto al dilema entre medioambiente y crecimiento económico porque es una región con economías en desarrollo, así como particularmente vulnerable a desastres naturales y con un nivel extremo de preocupación por el calentamiento global (Baez et al., 2017; Feng, Krueger y Oppenheimer, 2010; Pugatch, 2019; Rubin y Rossing, 2012). El apartado empírico del estudio descansa sobre dos tipos de pruebas estadísticas: modelos jerárquicos lineales y ecuaciones estructurales generalizadas. Los resultados muestran que la preocupación por las consecuencias del cambio climático tiene un efecto positivo sobre la priorización del medioambiente a expensas del crecimiento económico dependiendo de la situación económica de los individuos: las condiciones económicas adversas se asocian negativamente a la priorización del medioambiente para todos los niveles de preocupación por el calentamiento global. Solo cuando la situación económica individual mejora, la preocupación alta por los efectos del cambio climático se asocia a una mayor priorización del medioambiente que la ausencia de preocupación.

Este artículo hace dos contribuciones a la creciente investigación sobre las preferencias con respecto al dilema entre priorizar el medioambiente o el crecimiento económico. En primer lugar, analiza los efectos condicionantes y condicionados de la situación económica sobre la relación entre la preocupación por el cambio climático y las preferencias ambientales de los ciudadanos. Esto constituye un aporte a la vasta literatura sobre el rol de los factores económicos en la formación de actitudes sobre el

3 Disponible en: <https://www.vanderbilt.edu/lapop/>. Accesado el: fev. 2021. 
medioambiente que no ha explorado la discrepancia señalada entre percepciones en torno al cambio climático y actitudes ambientales. En segundo lugar, aporta al conocimiento de las actitudes medioambientales en la población de América Latina, una región que ha sido menos estudiada por la investigación sobre el tema. Comprender las creencias acerca del cambio climático y el apoyo público o la hostilidad hacia la priorización del medioambiente es importante al menos por dos razones: primero, en los sistemas democráticos, la opinión pública contribuye a dar forma a las políticas, incluidas las políticas de protección ambiental (Anderson, Bohmelt y Ward, 2017; Burstein, 2003; Page y Shapiro, 1983); y segundo, el éxito de la implementación de políticas proambientales depende en parte del consentimiento de los ciudadanos (Arpad, 2018; Basto-Abreu et al., 2016; Tjernstrom y Tietenberg, 2008).

El artículo está organizado de la siguiente manera. La primera sección, "La preocupación por el cambio climático y priorización del medioambiente", hace una revisión de las investigaciones recientes acerca de las creencias sobre el cambio climático y el medioambiente, y plantea el problema de investigación discutiendo la evidencia acerca de la opinión pública sobre cambio climático en América Latina. Una segunda sección, "Condiciones económicas, preocupación por el cambio climático y priorización del medioambiente", desarrolla el argumento acerca del efecto moderador de los constreñimientos económicos individuales sobre la relación entre preocupación por el cambio climático y la opción entre priorizar el medioambiente o el crecimiento económico. A continuación, en "Datos y métodos", se describen los datos y métodos utilizados en el análisis empírico. "Resultados y discusión" presenta los resultados del análisis empírico. El artículo termina con "Conclusiones".

\section{La preocupación por el cambio climático y priorización del medioambiente}

La opinión pública acerca del cambio climático es heterogénea con respecto a una serie de atributos: la ocurrencia del calentamiento global, su origen en actividades humanas como el uso de combustibles fósiles o en causas naturales no vinculadas a la actividad humana (Hamilton et al., 2015), la gravedad de sus consecuencias, el apoyo a las políticas de mitigación y la disposición a hacer cambios de comportamiento (Dunlap y York, 2008; Stokes, Wike y Carle, 2015).

El cambio climático es un fenómeno complejo y la mayoría de los ciudadanos carece de la preparación para informarse con la literatura científica. Como para otros fenómenos complejos, los ciudadanos usan la heurística para formar su opinión tomando las señales proporcionadas por su partido político preferido, o la información proveniente de medios de comunicación, organizaciones de la sociedad civil u otros formadores de opinión que les resultan confiables o con los que tienen afinidad ideológica (Guber, 2017; Leiserowitz, 2006; Nisbet, 2009).

La investigación acerca de la opinión pública sobre cambio climático ha mostrado que la preocupación por sus consecuencias, la percepción de riesgos para uno mismo y 
para la sociedad, son factores cruciales para explicar las actitudes ambientales de los ciudadanos y su disposición a incorporar cambios en el comportamiento, así como su apoyo a políticas públicas de mitigación y de protección del medioambiente.

El conocimiento y la preocupación por el cambio climático se asocian al apoyo de los ciudadanos a políticas de reducción de las emisiones de gases de efecto invernadero (O'Connor et al., 2002) y a su disposición a introducir cambios de comportamiento y de hábitos de consumo (Drews y Van Den Bergh, 2016; Leiserowitz, 2006; Viscusi y Zeckhauser, 2006). Las personas que perciben el riesgo de eventos ambientales adversos son más propensas a apoyar políticas para prevenirlos y mitigarlos, incluso si estos comportamiento y políticas implican costos, y a renunciar a cierto bienestar (Hersch y Viscusi, 2006; Slovic, 1987). Zahran y coautores (2006) analizan los efectos de la vulnerabilidad a riesgos objetivos y de la percepción subjetiva de riesgos del cambio climático y encuentran que la percepción subjetiva del riesgo se asocia al apoyo a políticas de mitigación y a medidas gubernamentales costosas. En este mismo sentido, un estudio realizado en Malta muestra que la percepción de que el cambio climático puede poner en riesgo la vida, agravar la escasez de agua, causar enfermedades y reducir el nivel de vida en general constituye un impulso determinante para el apoyo de la población a las políticas de mitigación (Debono, Vincenti y Calleja, 2010). Por el contrario, el déficit de preocupación por los temas ambientales es un claro depresor del apoyo a las políticas para mitigar las consecuencias del calentamiento global (Lorenzoni, Nicholson-Cole y Whitmarsh, 2007; Mildenberger y Tingley, 2019).

La preocupación por las consecuencias del calentamiento global también se asocia a la disposición de los ciudadanos a hacer cambios de comportamiento que implican costos individuales o para el hogar. Los ciudadanos con un nivel alto de preocupación por las consecuencias del calentamiento global tienen más probabilidades de introducir cambios en su consumo de energía. Por ejemplo, están más dispuestos a reducir el uso de calefacción o de aire acondicionado, y a cambiar los electrodomésticos por unos más eficientes (O'Connor, Bord y Fisher, 1999). Asimismo, quienes tienen una mayor percepción subjetiva de la gravedad de los impactos del cambio climático tienen una disposición mayor a pagar para compensar los daños ocasionados por su parte de responsabilidad en las emisiones (Brouwer, Brander y Van Beukering, 2008; Sonnenschein y Smedby, 2019; Streimikiene et al., 2019) y para implementar políticas que reduzcan futuras muertes relacionadas con el cambio climático (Graham et al., 2019).

Las asociaciones descriptas entre las actitudes hacia el calentamiento global y las preferencias ambientales deberían encontrar apoyo empírico en América Latina, cuyos ciudadanos tienen en promedio el nivel de preocupación por las consecuencias del cambio climático más alto del mundo. Sin embargo, como veremos a continuación, esto no ocurre. En 2015, la Encuesta Global de Actitudes del Pew Research Center encontró que el 74 por ciento de los encuestados en América Latina consideraba que el cambio climático tendría consecuencias serias. En contraste, en Europa y en Estados Unidos el porcentaje de ciudadanos preocupados por el cambio climático es de 54 por ciento y 18 por ciento 
respectivamente (Stokes, Wike y Carle, 2015). En este mismo sentido, la encuesta del Barómetro de las Américas de 2016 registra que un 77 por ciento de los encuestados considera que las consecuencias del cambio climático serán serias en su país. Este alto nivel de preocupación por el cambio climático en América Latina no siempre se asocia a la priorización del medioambiente. La Figura 1 muestra que, entre quienes consideran que las consecuencias del cambio climático serán serias, un $22 \%$ no cree que se deba priorizar el medioambiente y prefiere priorizar el crecimiento económico.

Figura 1

Preocupación por las consecuencias del cambio climático y prioridades entre medioambiente y crecimiento económico en América Latina

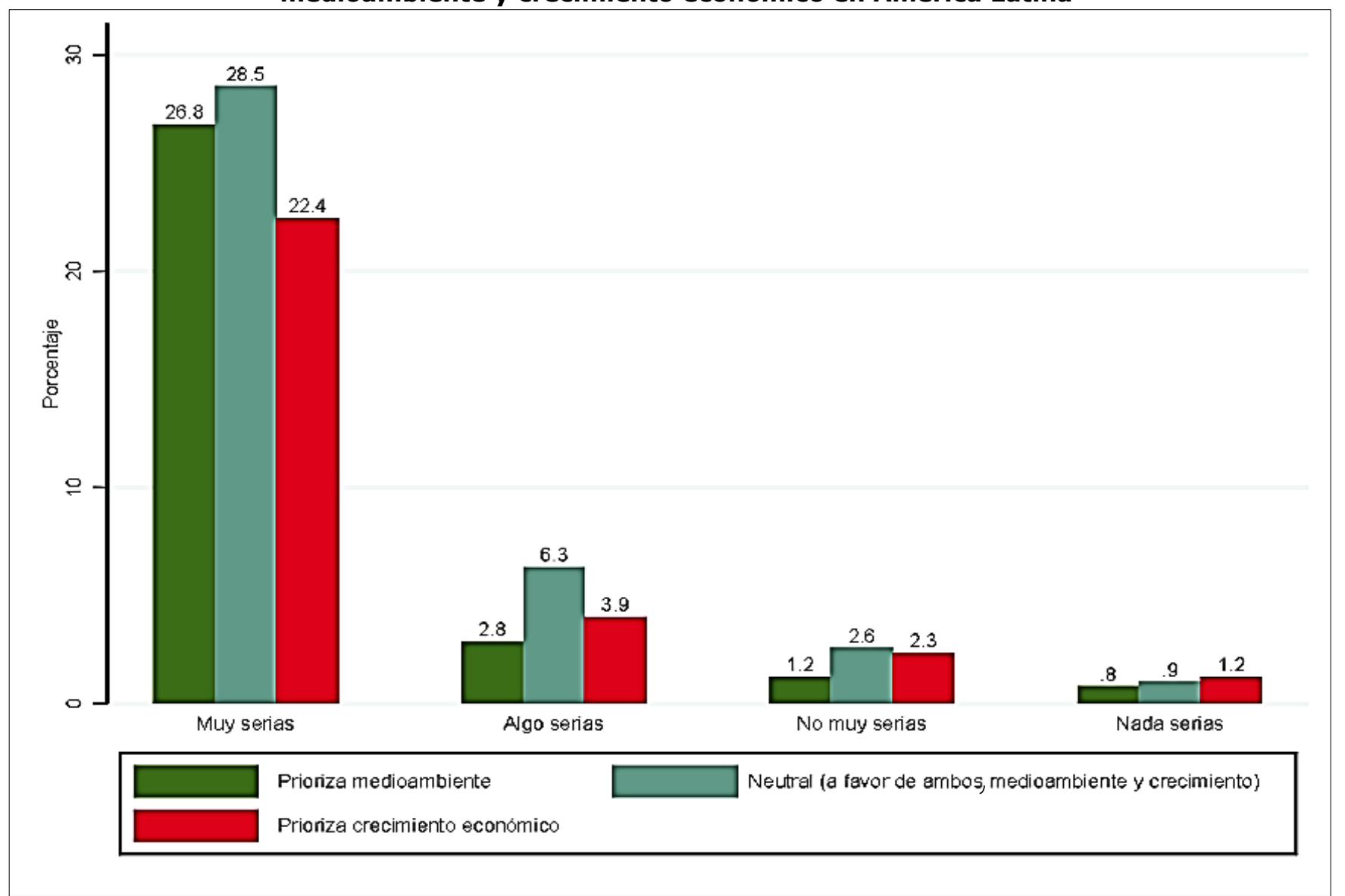

Fuente: Elaboración propia con datos de Lapop 2016.

Porcentajes de respuestas a las preguntas: "ENV2B1. Si no se hace nada para reducir el cambio climático en el futuro, ¿qué tan serio piensa usted que sería el problema para (país)?" Y "ENV1C1. Alguna gente cree que hay que priorizar la protección del medioambiente sobre el crecimiento económico, mientras otros creen que el crecimiento económico debería priorizarse sobre la protección ambiental. En una escala de 1 a 7 en la que 1 significa que el medioambiente debe ser la principal prioridad, y 7 significa que el crecimiento económico debe ser la principal prioridad, ¿dónde se ubicaría usted?". Recodifico la variable original agrupando las respuestas en tres categorías: "Prioriza medioambiente" (valores 1 y 2), "Neutral, a favor de ambos, medioambiente y crecimiento" (valores 3,4 y 5 ) y "Prioriza crecimiento económico" (valores 6 y 7 ). 
¿Por qué la preocupación por el cambio climático no siempre conduce a priorizar la protección del medioambiente ${ }^{4}$ ¿Bajo qué condiciones la percepción de consecuencias graves del calentamiento global conduce a los ciudadanos a priorizar el medioambiente sobre el crecimiento? El siguiente apartado responde a esta pregunta retomando las explicaciones económicas para las preferencias ambientales y argumenta que los constreñimientos materiales condicionan la relación entre las actitudes hacia el cambio climático y las preferencias frente al dilema entre priorizar el medioambiente o el crecimiento económico.

\section{Condiciones económicas, preocupación por el cambio climático y priorización del medioambiente}

Desde la década de 1970, los científicos sociales han argumentado que es necesario centrarse en los procesos económicos para comprender el activismo ambiental y las actitudes hacia el medioambiente (Inglehart, 1977). La vasta producción académica que ha abordado esta relación dio lugar a dos teorías: la teoría de los valores postmaterialistas y la teoría de la riqueza.

Desde la ciencia política, la tesis de los valores posmaterialistas de Inglehart (1977) argumenta que los valores de los individuos cambian de acuerdo con el nivel de bienestar material. A medida que los países se desarrollan y las sociedades se hacen más ricas, sus ciudadanos van abandonando su preocupación por temas materiales para dar lugar a intereses en torno a la protección del medioambiente, así como a la expansión de las libertades individuales y políticas y al reconocimiento de la diversidad. Este cambio cultural es resultado del reemplazo gradual de generaciones materialistas, conformadas por individuos nacidos y criados en contextos de mayor privación material, por nuevas generaciones más jóvenes con valores postmaterialistas cuyos integrantes nacieron y se criaron en contextos de abundancia material (Inglehart, 1981, 1985; Inglehart y Abramson, 1994).

El argumento de los valores postmaterialistas ha dado lugar a un debate amplio. Por una parte, algunos estudios muestran que los ciudadanos pobres de los países desarrollados y los ciudadanos de los países pobres también están preocupados por el medioambiente (Stenner y Nwokora, 2015). Otros han argumentado que las explicaciones para la preocupación por el medioambiente en países con diferentes niveles de desarrollo serían heterogéneas, en los países ricos se explicaría por los valores postmaterialistas y

\footnotetext{
4 Los pocos estudios que han explorado la brecha entre preocupación por el cambio climático y actitudes medioambientales han encontrado que los ciudadanos no están dispuestos a cambiar su comportamiento para mitigar los efectos del calentamiento global a menos que el gobierno imponga regulaciones para obligarlos a actuar porque entienden que los problemas ambientales requieren respuestas colectivas (Darier y Schüle, 1999; Hinchliffe, 1996; Lorenzoni, Nicholson-Cole y Whitmarsh, 2007), o que se ven empujados a la inacción con respecto a los problemas ambientales porque la comprensión de la complejidad del cambio climático les produce un sentimiento de fatalismo (Mayer y Smith, 2019; Nisbet, 2009).
} 
en los pobres por las desigualdades ambientales que amenazan la salud y el bienestar (Brechin, 1999; Dunlap y Mertig, 1995, 1997; Dunlap y York, 2008; Inglehart, 1995).

Desde una perspectiva económica, se sostiene que la riqueza tiene un efecto positivo sobre la protección del medioambiente con el siguiente razonamiento económico. La protección del medioambiente, o la restauración de un medioambiente dañado, constituye un bien "superior", y su demanda se incrementa a medida que el ingreso crece (Bakaki y Bernauer, 2018; Kachi, Bernauer y Gampfer, 2015). Para los ciudadanos, dedicar recursos a la protección del medioambiente implica el costo de oportunidad de renunciar al consumo de otros bienes. A nivel agregado, se ha comprobado una relación positiva entre la riqueza de un país y su nivel de apoyo a la protección del medioambiente porque los países ricos pueden asumir el costo de oportunidad (Diekmann y Franzen, 1999; Dunlap y York, 2008; Franzen, 2003; Franzen y Vogl, 2013; Gelissen, 2007). Las naciones más ricas tienen más recursos para dedicar al activismo ambiental, y sus ciudadanos tienen más probabilidades de interesarse en la protección del medioambiente. Por el contrario, los países menos industrializados tienen más interés en priorizar el crecimiento económico porque grandes porciones de su población aún carecen de acceso a una seguridad material básica. En esta línea, la hipótesis de la curva ambiental de Kuznets (EKC) (Kuznets, 1955) sugiere que la protección del medioambiente se deteriora en las primeras etapas del desarrollo económico y mejora en etapas posteriores, dibujando una curva en forma de $U$ (Dinda, 2004). Cuando un país en proceso de industrialización alcanza cierto nivel de desarrollo económico, el progreso tecnológico y las crecientes actitudes proambientales de los ciudadanos conducen a niveles más altos de protección ambiental y a un ciclo virtuoso de crecimiento con protección ambiental (Dinda, 2004). Por su parte, los países pobres o menos industrializados esperan una mayor responsabilidad ambiental por parte de los más desarrollados (Bou-Habib, 2019; Mittiga, 2019; Neumayer, 2000).

A nivel individual, la preocupación ambiental de los ciudadanos se desarrolla y tiene influencia en la protección del medioambiente una vez que los individuos y el país han alcanzado un cierto nivel de desarrollo económico (Ficko y Boncina, 2019; Franzen y Meyer, 2009); son las personas más ricas quienes están más dispuestas a renunciar a parte de sus ingresos a cambio de un medioambiente más limpio (Diekmann y Franzen, 1999; Franzen y Meyer, 2009). Por ejemplo, Franzen y Vogl (2012) analizan los datos de tres encuestas - Programa Internacional de Encuestas Sociales (ISSP), Encuesta Mundial de Valores (WVS) y Estudio Europeo de Valores (EVS) - y muestran que la riqueza tiene un efecto positivo sobre la disposición de los individuos a priorizar el medioambiente y a pagar por su protección (Franzen, 2003).

En resumen, un cuerpo amplio de investigaciones ha mostrado que la percepción subjetiva de los riesgos del cambio climático y la preocupación por las posibles consecuencias se asocian a actitudes a favor del medioambiente, a la disposición a cambiar comportamientos y al apoyo a políticas de protección del medioambiente. Sin embargo, para que la preocupación por las consecuencias del cambio climático moldee las preferencias relativas al dilema entre priorizar el medioambiente o el crecimiento 
PREOCUPACIÓN POR EL CAMBIO CLIMÁTICO, CONDICIONES ECONÓMICAS INDIVIDUALES Y PRIORIZACIÓN DEL MEDIOAMBIENTE..

económico, los individuos deben encontrarse en condiciones económicas que les permitan optar entre priorizar el medioambiente y renunciar a avanzar en la consecución de mayores niveles de bienestar. Las condiciones económicas individuales adversas de los ciudadanos actúan como constreñimientos que limitan las opciones frente al dilema entre medioambiente y crecimiento y socavan los efectos positivos de la preocupación por el calentamiento global sobre la posible priorización del medioambiente porque no están en condiciones de renunciar al crecimiento económico. En cambio, las condiciones económicas individuales aventajadas permiten a quienes perciben los riesgos del calentamiento global elegir priorizar el medioambiente. Por consiguiente, espero que el efecto de la percepción subjetiva de las consecuencias del cambio climático sobre las preferencias entre priorizar el medioambiente o el crecimiento económico sea moderado por la situación económica individual de los ciudadanos. Esto conduce a las siguientes hipótesis:

H1: La preocupación por las consecuencias del cambio climático se asocia a la priorización del medioambiente sobre el crecimiento económico, dependiendo de la situación económica individual. La preocupación por el calentamiento global conduce a la priorización del medioambiente cuando la situación económica individual es aventajada.

H2: La situación económica individual se asocia a la priorización del medioambiente dependiendo del nivel de preocupación por el cambio climático. La situación económica individual favorable conduce a una mayor priorización del medioambiente entre los ciudadanos con alta preocupación por el cambio climático que entre los ciudadanos con menor preocupación.

Con la primera hipótesis espero que la alta preocupación por el cambio climático se asocie a una priorización del medioambiente cuando los ciudadanos disfrutan de una situación económica individual favorable. La segunda hipótesis se refiere al efecto condicionado de la situación económica sobre las preferencias relativas al medioambiente dependiendo del nivel de percepción del riesgo ambiental. La relación conocida entre las condiciones económicas de los ciudadanos y la priorización del medioambiente según la cual la situación económica tiene un efecto positivo sobre la priorización del medioambiente a expensas del desarrollo económico es moderada por el nivel de preocupación por el cambio climático. Entre los ciudadanos con mejor situación económica individual, espero que el efecto de la economía sobre la priorización del medioambiente sea mayor para los que consideran que estas consecuencias pueden ser serias que para quienes tienen menor nivel de preocupación por las posibles consecuencias del calentamiento global.

\section{Datos y métodos}

Para poner a prueba las hipótesis propuestas, utilizo datos de las encuestas del Barómetro de las Américas (Lapop) de 18 países latinoamericanos. Desde 2004, el Barómetro de las Américas es un proyecto de encuestas bianuales que emplea cuestionarios estandarizados y muestras probabilísticas nacionales para investigar las actitudes y experiencias políticas de los ciudadanos en edad de votar en los países de 
América. Utilizo las encuestas administradas en 2016 porque los cuestionarios de este año incluyen preguntas sobre la preocupación con respecto al cambio climático y la priorización del medioambiente o el crecimiento económico para todos los países. Los datos para este documento consisten en 26.423 respuestas de encuestas realizadas en los siguientes países: Argentina, Bolivia, Brasil, Chile, Colombia, Costa Rica, República Dominicana, Ecuador, El Salvador, Guatemala, Honduras México, Nicaragua, Panamá, Paraguay, Perú, Uruguay y Venezuela.

\section{Variables dependiente e independientes}

La variable dependiente proviene de la siguiente pregunta de la encuesta de Lapop, diseñada para medir las preferencias de los ciudadanos frente a la disyuntiva entre priorizar el medioambiente o el crecimiento económico: "ENV1. Alguna gente cree que hay que priorizar la protección del medioambiente sobre el crecimiento económico, mientras otros creen que el crecimiento económico debería priorizarse sobre la protección ambiental. En una escala de 1 a 7 en la que 1 significa que el medioambiente debe ser la principal prioridad, y 7 significa que el crecimiento económico debe ser la principal prioridad, ¿dónde se ubicaría usted?". Para facilitar la interpretación de la preferencia de priorizar el medioambiente he invertido la escala, de modo que 1 refleja la priorización del crecimiento económico y 7 la priorización del medioambiente.

Las variables independientes son dos. La "Preocupación por el cambio climático", medida a través de la siguiente pregunta: "ENV2B1. Si no se hace nada para reducir el cambio climático en el futuro, ¿qué tan serio piensa usted que sería el problema para (País)?". Las opciones de respuesta son: (1) Muy serio, (2) Algo serio, (3) Poco serio y (4) Nada serio. Para esta variable también he invertido la escala para facilitar la interpretación.

Para medir la segunda variable independiente, la "Situación económica individual", sigo la práctica estándar en los estudios de los países en desarrollo utilizando un índice de riqueza patrimonial del hogar agregando la presencia de bienes de consumo en el hogar del encuestado. Las medidas de bienestar basadas en la propiedad de los activos del hogar están menos sujetas a fluctuaciones de ingresos a corto plazo y tienen menos casos perdidos por falta de respuesta (Baldwin y Huber, 2010). El índice de riqueza resulta de un análisis de componentes principales con respuestas a preguntas sobre la presencia de los siguientes elementos: televisión, refrigerador, teléfono convencional, teléfono celular, vehículo/automóvil, lavadora, horno de microondas, motocicleta, plomería interior, baño interior, y computadora.

\section{Variables de control}

Los modelos controlan por los posibles efectos de confusión de variables relacionadas con la percepción del riesgo ambiental y con las preferencias ambientales, incluyendo variables políticas como la ideología (Dunlap y McCright, 2008; Harring, Jagers 
y Matti, 2017; McCright y Dunlap, 2013), la evaluación de la situación económica personal y nacional, y la eficacia política e individual (Arnold, 2012; Kolln, 2018; Lergetporer et al., 2018). Adicionalmente, se incluyen controles socio-demográficos que estudios previos han asociado a actitudes medioambientales: edad (Hersch y Viscusi, 2006; Johnson y Schwadel, 2019), género, presencia de hijos en el hogar (McCright y Xiao, 2014), años de educación (Chankrajang y Muttarak, 2017; Post y Meng, 2018), y residencia urbana o rural (Borisova et al., 2018).

Para medir la evaluación de la situación económica personal uso las respuestas a la siguiente pregunta: "IDIO2. Considera usted que su situación económica actual es ¿mejor, igual o peor que la de hace doce meses?". Y mido la evaluación de la situación económica nacional usando la pregunta "SOCT2. ¿Considera usted que la situación económica del país es mejor, igual o peor que hace doce meses?".

Considerando que la eficacia política y personal estarían asociadas positivamente a las actitudes favorables al medioambiente (Kellstedt, Zahran y Vedlitz, 2008), incluyo las respuestas a las siguientes preguntas. Una primera que captura la eficacia política: "EFF1. A los que gobiernan el país les interesa lo que piensa la gente como usted. ¿Hasta qué punto está de acuerdo o en desacuerdo con esta frase?". Y una segunda pregunta para medir la eficacia personal: "EFF2. Usted siente que entiende bien los asuntos políticos más importantes del país. ¿Hasta qué punto está de acuerdo o en desacuerdo con esta frase?" Ambas preguntas se responden en una escala de 1 a 7.

La ideología de izquierda o derecha, que ciertamente está ligada a las actitudes sobre la redistribución de la riqueza, no está tan obviamente vinculada a las preferencias medioambientales. Sin embargo, los estudios muestran quienes prefieren un gobierno más pequeño y una economía de libre mercado también favorecen el crecimiento económico en lugar de la protección del medioambiente (Neumayer, 2004). Esta asociación probablemente sea el resultado de que los ciudadanos tomen en cuenta las posiciones de los partidos con los que tienen afinidad política como atajo informativo para formar su opinión sobre temas ambientales (Malka y Lelkes, 2010). Los partidos de centroizquierda tienen una plataforma que recupera problemas medioambientales (Neumayer, 2004), y los partidos de centroderecha priorizan el desarrollo a expensas del medioambiente. Por ese motivo, se ha argumentado que es posible esperar que quienes se ubican a la izquierda del espectro ideológico sean más favorables a priorizar el medioambiente que quienes se ubican a la derecha (Kvaloy, Finseraas y Listhaug, 2012). Para controlar por la ideología incluyo las respuestas a la siguiente pregunta "L1. Cambiando de tema, en esta tarjeta tenemos una escala del 1 a 10 que va de izquierda a derecha, en la que el 1 significa izquierda y el 10 significa derecha. Hoy en día cuando se habla de tendencias políticas, mucha gente habla de aquellos que simpatizan más con la izquierda o con la derecha. Según el sentido que tengan para usted los términos 'izquierda' y 'derecha' cuando piensa sobre su punto de vista político, ¿dónde se encontraría usted en esta escala?".

Los medios de comunicación pueden desempeñar un papel importante en la configuración de las actitudes hacia el medioambiente, en oposición al crecimiento 
económico, incluso entre los menos interesados (Elias et al., 2019; Happer y Wellesley, 2019; Ostman y Parker, 1987). Controlo por el nivel de exposición incluyendo respuestas a la siguiente pregunta: "GI0. ¿Con qué frecuencia sigue las noticias, ya sea en la televisión, la radio, los periódicos o el Internet? (1) Diariamente, (2) Algunas veces a la semana, (3) Algunas veces al mes, (4) Rara vez, (5) Nunca".

Los modelos controlan por la presencia de niños en el hogar. Por esas razones, las personas de hogares con niños pueden valorar más los beneficios de un medioambiente protegido (Lubell et al., 2006).

A nivel de país, los modelos controlan por el nivel de democracia, medido por el índice Polity2, el nivel de desarrollo capturado por el PIB per cápita, la dependencia de los recursos naturales medida como la suma de las rentas del petróleo, el gas natural, el carbón, los minerales y bosques como parte del PIB. Se puede esperar que los encuestados prioricen el crecimiento económico y el empleo sobre el medioambiente si viven en países más pobres o dependientes de los recursos naturales. Las democracias han demostrado una posición más fuerte en la mitigación del cambio climático que los regímenes no democráticos; cooperan en tratados ambientales internacionales y adoptan políticas ambientales más estrictas (Cao y Ward, 2015; Povitkina, 2018; Ward, 2008; Wurster, 2013).

\section{Método}

Para poner a prueba las hipótesis propuestas implemento dos estrategias: modelos de regresión jerárquica lineal y modelos de elección discreta multinomial. Los modelos jerárquicos lineales toman en cuenta que las observaciones a nivel individual están anidadas en contextos nacionales y capturan adecuadamente esta estructura de los datos (Raudenbush y Bryk, 2002; Snijders y Bosker, 1999). Especifico un modelo jerárquico lineal de intercepción aleatoria que evalúa el efecto de la situación económica sobre la relación entre la preocupación por el cambio climático y la priorización del medioambiente o el crecimiento, al tiempo que controla los factores de confusión a nivel individual y a nivel nacional. En estos modelos, las intercepciones específicas de cada país capturan la idea de que las personas pueden tener diferentes niveles de apoyo a la priorización del medioambiente en diferentes países, aunque tengan características similares, es decir, los mismos valores en los predictores a nivel individual de actitudes proambientales. Este tipo de análisis nos permite explorar las asociaciones generales, teniendo en cuenta que los países difieren con respecto a sus medias. Los datos de la encuesta del Barómetro de las Américas emplean un diseño de muestra complejo, que incluye estratificación, agrupamiento y ponderación de algunas observaciones; por ello, todos los análisis tienen en cuenta estos elementos de diseño al estimar los modelos.

En segundo lugar, como pruebas adicionales, especifico modelos multinomiales para variables de elección discreta. Recordemos que la variable dependiente principal utilizada en este estudio proviene de respuestas a la pregunta de la encuesta de Lapop 
PREOCUPACIÓN POR EL CAMBIO CLIMÁTICO, CONDICIONES ECONÓMICAS INDIVIDUALES Y PRIORIZACIÓN DEL MEDIOAMBIENTE...

ENV1, que aborda las preferencias entre priorizar el medioambiente a expensas del desarrollo económico o viceversa. La pregunta admite múltiples respuestas (entre 1 y 7), lo que la convierte en una opción natural para un análisis empírico de elección discreta multinomial. Para especificar estos modelos, recodifico la variable original agrupando las respuestas en tres categorías: "A favor del medioambiente" (valores 1 y 2), "Neutral, a favor de ambos medioambiente y crecimiento" (valores 3, 4 y 5) y "A favor del crecimiento" (valores 6 y 7). Para examinar empíricamente en qué medida la preferencia por priorizar el medioambiente - o el crecimiento económico, o ambos - está relacionada sistemáticamente con el nivel de preocupación por el cambio climático condicionado por la situación económica individual, como una alternativa respecto de las otras opciones, he especificado un modelo de ecuaciones estructurales generalizadas para variable dependiente categórica multinomial y una estructura de datos anidados. Los modelos multinomiales no pueden estimar el nivel de los coeficientes directamente porque las opciones observadas solo contienen información sobre preferencias relativas. Por lo tanto, no podemos distinguir los efectos absolutos en la elección de las actitudes proambientales, sino solo el grado en que cada variable explicativa hace que un encuestado sea más o menos proclive a priorizar el medioambiente o el crecimiento económico.

\section{Resultados y discusión}

La Tabla 1 reporta las distribuciones de frecuencias de las respuestas a las preguntas para medir la preocupación por el cambio climático y la priorización del medioambiente o el crecimiento económico. En todos los países, con excepción de Paraguay, más del 70 por ciento de los encuestados considera que las consecuencias del cambio climático serán graves, y en promedio un tercio de los encuestados se inclina por priorizar el medioambiente a expensas del crecimiento económico. 
Tabla 1

Preocupación por el cambio climático y prioridades entre medioambiente y crecimiento económico en los países de América Latina 2016

\begin{tabular}{|c|c|c|c|c|c|c|c|}
\hline \multirow[b]{2}{*}{ País } & \multicolumn{4}{|c|}{ Preocupación por el cambio climático } & \multicolumn{3}{|c|}{$\begin{array}{c}\text { Prioridades: medioambiente o crecimiento } \\
\text { económico }\end{array}$} \\
\hline & Muy seria & Algo seria & $\begin{array}{l}\text { No muy } \\
\text { seria }\end{array}$ & $\begin{array}{l}\text { Nada } \\
\text { seria }\end{array}$ & $\begin{array}{c}\text { Pro- } \\
\text { medioambiente }\end{array}$ & Ambos & $\begin{array}{c}\text { Pro- } \\
\text { crecimiento } \\
\text { económico }\end{array}$ \\
\hline Argentina & 72,8 & 19,07 & 5,07 & 3,07 & 32,64 & 47,57 & 19,79 \\
\hline Bolivia & 73,66 & 16,74 & 7,31 & 2,3 & 35,59 & 36,8 & 27,61 \\
\hline Brasil & 80,04 & 9,82 & 6,26 & 3,89 & 30,51 & 40,09 & 29,4 \\
\hline Chile & 77,37 & 14,46 & 6,05 & 2,12 & 36,25 & 44,86 & 18,9 \\
\hline Colombia & 78,86 & 10,96 & 6,55 & 3,63 & 40,32 & 37,86 & 21,81 \\
\hline Costa Rica & 81,29 & 12,72 & 4,86 & 1,13 & 20,75 & 49 & 30,25 \\
\hline $\begin{array}{l}\text { República } \\
\text { Dominicana }\end{array}$ & 78,93 & 13,02 & 3,49 & 4,56 & 28,7 & 26,76 & 44,54 \\
\hline Ecuador & 73,27 & 17,58 & 6,86 & 2,29 & 30,09 & 39,5 & 30,41 \\
\hline El Salvador & 84,63 & 7,07 & 5,58 & 2,72 & 24,35 & 37,44 & 38,21 \\
\hline Guatemala & 84,89 & 6,24 & 5,32 & 3,55 & 30,74 & 33,22 & 36,04 \\
\hline Honduras & 80,63 & 9,33 & 6,67 & 3,37 & 28,77 & 29,75 & 41,49 \\
\hline México & 77,58 & 14,06 & 6,09 & 2,27 & 29,6 & 44,01 & 26,39 \\
\hline Nicaragua & 83,08 & 9,98 & 4,73 & 2,2 & 33,33 & 28,2 & 38,47 \\
\hline Panamá & 78,61 & 12,61 & 4,42 & 4,36 & 37,66 & 31,14 & 31,2 \\
\hline Paraguay & 67,19 & 14,82 & 13,04 & 4,94 & 35,77 & 36,24 & 27,99 \\
\hline Perú & 75,77 & 13,26 & 7,73 & 3,24 & 32,97 & 39,97 & 27,07 \\
\hline Uruguay & 73,74 & 20,01 & 4,52 & 1,73 & 30,76 & 51,07 & 18,18 \\
\hline Venezuela & 75,81 & 15,97 & 4,89 & 3,32 & 26,67 & 36,92 & 36,4 \\
\hline Total & 77,62 & 13,21 & 6,14 & 3,03 & 31,51 & 38,41 & 30.08 \\
\hline \multicolumn{8}{|c|}{$\begin{array}{l}\text { ENV2B1. Si no se hace nada para reducir el cambio climático en el futuro, ¿qué tan serio piensa usted que sería el } \\
\text { problema para (país)? } \\
\text { ENV1C1. Alguna gente cree que hay que priorizar la protección del medioambiente sobre el crecimiento económico, } \\
\text { mientras otros creen que el crecimiento económico debería priorizarse sobre la protección ambiental. En una escala } \\
\text { de } 1 \text { a } 7 \text { en la que } 1 \text { significa que el medioambiente debe ser la principal prioridad, y } 7 \text { significa que el crecimiento } \\
\text { económico debe ser la principal prioridad, ¿dónde se ubicaría usted? }\end{array}$} \\
\hline
\end{tabular}

Fuente: Elaboración propia con datos de Lapop 2016.

La Tabla 2 presenta los resultados de los modelos jerárquicos lineales para poner a prueba las hipótesis acerca del efecto condicionado de la preocupación por las consecuencias del cambio climático sobre la priorización del medioambiente. El Modelo 1 consiste en una especificación sin interacción y sin controles. El Modelo 2 incluye la interacción entre el indicador de la situación económica individual, el índice de riqueza patrimonial del hogar y las categorías para cada nivel de preocupación por el cambio climático con la finalidad de poner a prueba que el efecto de las creencias acerca de la 
PREOCUPACIÓN POR EL CAMBIO CLIMÁTICO, CONDICIONES ECONÓMICAS INDIVIDUALES Y PRIORIZACIÓN DEL MEDIOAMBIENTE...

gravedad del cambio climático sobre las actitudes frente al dilema entre medioambiente y crecimiento es moderado por la situación económica. Los modelos 3 y 4 incluyen los mismos términos de interacción incorporando controles a nivel individual (Modelo 3) y a nivel nacional (Modelo 4).

¿En qué condiciones podemos esperar un impacto positivo de la preocupación por las consecuencias del cambio climático sobre la priorización del medioambiente? Los resultados reportados en la columna 1 sugieren que, en promedio, la alta preocupación por las consecuencias del calentamiento global (consecuencias serias) y la riqueza tienen un impacto positivo sobre la priorización del medioambiente. Dado que las hipótesis de este trabajo se refieren a efectos condicionados, los resultados centrales provienen de los modelos 2,3 y 4 , que incluyen el término de interacción entre riqueza patrimonial y los distintos niveles de preocupación por el cambio climático. Los coeficientes negativos para los distintos niveles de preocupación por el cambio climático indican que, entre los encuestados con una situación económica desfavorable, la preocupación por el cambio climático tiene siempre un efecto negativo sobre la priorización del medioambiente. Sin embargo, los coeficientes positivos y significativos para los términos de interacción entre riqueza patrimonial y las categorías para los distintos niveles de preocupación por el cambio climático sugieren que, a medida que la riqueza patrimonial crece, la preocupación por el calentamiento global se asocia positivamente a la priorización del medioambiente. Las personas ricas que consideran que las consecuencias del cambio climático serán muy serias priorizan el medioambiente más que las personas pobres con el mismo nivel de preocupación por el riesgo ambiental.

En cuanto a las variables de control, los resultados de la Tabla 2 muestran que la mayoría de las variables políticas y sociodemográficas se comportan según lo esperado por el conocimiento existente. Los encuestados que se ubican hacia la derecha del espectro ideológico, quienes siguen los medios con más frecuencia, las mujeres y los residentes urbanos priorizan el medioambiente en menor medida que el crecimiento económico; mientras que ser joven, más educado y con más eficacia personal se asocia a la priorización del medioambiente. Contra las expectativas de la literatura, la eficacia política y la presencia de niños se relacionan negativamente con la priorización del medioambiente. Los resultados son casi idénticos en las tres especificaciones. 
Tabla 2

Preocupación sobre el cambio climático, situación económica y priorización del crecimiento económico en América Latina 2016

\begin{tabular}{|c|c|c|c|c|}
\hline & Modelo 1 & Modelo 2 & Modelo 3 & Modelo 4 \\
\hline $\begin{array}{l}\text { Consecuencias Cambio climático No muy } \\
\text { serias X Riqueza patrimonial }\end{array}$ & & $\begin{array}{c}0.079 * * \\
(0.035)\end{array}$ & $\begin{array}{c}0.077 * * \\
(0.037)\end{array}$ & $\begin{array}{l}0.084 * * \\
(0.038)\end{array}$ \\
\hline $\begin{array}{l}\text { Consecuencias Cambio climático Algo serias } \mathrm{X} \\
\text { Riqueza patrimonial }\end{array}$ & & $\begin{array}{l}0.139 * * * \\
(0.032)\end{array}$ & $\begin{array}{c}0.127 * * * \\
(0.034)\end{array}$ & $\begin{array}{c}0.129 * * * \\
(0.035)\end{array}$ \\
\hline $\begin{array}{l}\text { Consecuencias Cambio climático Muy serias } X \\
\text { Riqueza patrimonial }\end{array}$ & & $\begin{array}{c}0.163 * * * \\
(0.030)\end{array}$ & $\begin{array}{c}0.163 * * * \\
(0.031) \\
\end{array}$ & $\begin{array}{c}0.167 * * * \\
(0.032)\end{array}$ \\
\hline $\begin{array}{l}\text { Consecuencias Cambio climático No muy } \\
\text { serias }\end{array}$ & $\begin{array}{l}-0.130 \\
(0.097) \\
\end{array}$ & $\begin{array}{c}-0.677 * * \\
(0.269) \\
\end{array}$ & $\begin{array}{l}-0.765 * * * \\
(0.284) \\
\end{array}$ & $\begin{array}{c}-0.794 * * * \\
(0.290) \\
\end{array}$ \\
\hline Consecuencias Cambio climático Algo serias & $\begin{array}{c}0.114 \\
(0.088)\end{array}$ & $\begin{array}{l}-0.857 * * * \\
(0.248)\end{array}$ & $\begin{array}{l}-1.023 * * * \\
(0.262)\end{array}$ & $\begin{array}{l}-1.034 * * * \\
(0.268)\end{array}$ \\
\hline Consecuencias Cambio climático Muy serias & $\begin{array}{l}0.596 * * * \\
(0.081)\end{array}$ & $\begin{array}{c}-0.565^{* *} \\
(0.224) \\
\end{array}$ & $\begin{array}{l}-0.808 * * * \\
(0.237)\end{array}$ & $\begin{array}{c}-0.814 * * * \\
(0.243)\end{array}$ \\
\hline Riqueza patrimonial & $\begin{array}{c}0.095 * * * \\
(0.006)\end{array}$ & $\begin{array}{l}-0.054 * \\
(0.029) \\
\end{array}$ & $\begin{array}{c}-0.108 * * * \\
(0.031) \\
\end{array}$ & $\begin{array}{c}-0.115^{* * *} \\
(0.032) \\
\end{array}$ \\
\hline Eficacia política & & & $\begin{array}{c}-0.094 * * * \\
(0.007) \\
\end{array}$ & $\begin{array}{c}-0.096 * * * \\
(0.008)\end{array}$ \\
\hline Eficacia individual & & & $\begin{array}{c}0.001 \\
(0.009)\end{array}$ & $\begin{array}{c}0.005 \\
(0.009)\end{array}$ \\
\hline Situación económica nacional = Igual & & & $\begin{array}{c}0.121 * * \\
(0.052)\end{array}$ & $\begin{array}{c}0.122 * * \\
(0.052)\end{array}$ \\
\hline Situación económica nacional $=$ Peor & & & $\begin{array}{c}0.005 \\
(0.052) \\
\end{array}$ & $\begin{array}{c}0.005 \\
(0.052) \\
\end{array}$ \\
\hline Situación económica personal = Igual & & & $\begin{array}{c}0.110 * * * \\
(0.041)\end{array}$ & $\begin{array}{c}0.121 * * * \\
(0.041)\end{array}$ \\
\hline Situación económica personal = Peor & & & $\begin{array}{c}0.062 \\
(0.044)\end{array}$ & $\begin{array}{l}0.081 * \\
(0.045)\end{array}$ \\
\hline Ideología & & & $\begin{array}{c}-0.046 * * * \\
(0.005)\end{array}$ & $\begin{array}{c}-0.044 * * * \\
(0.005)\end{array}$ \\
\hline Atención a medios & & & $\begin{array}{c}-0.015 \\
(0.014) \\
\end{array}$ & $\begin{array}{c}-0.017 \\
(0.015) \\
\end{array}$ \\
\hline Educación & & & $\begin{array}{c}0.082 * * * \\
(0.004)\end{array}$ & $\begin{array}{c}0.082 * * * \\
(0.004)\end{array}$ \\
\hline Niños en el hogar & & & $\begin{array}{c}-0.117^{* * *} \\
(0.036)\end{array}$ & $\begin{array}{c}-0.118 * * * \\
(0.037)\end{array}$ \\
\hline Mujer & & & $\begin{array}{c}-0.191 * * * \\
(0.029) \\
\end{array}$ & $\begin{array}{c}-0.179 * * * \\
(0.030)\end{array}$ \\
\hline Edad & & & $\begin{array}{c}-0.010 * * * \\
(0.001)\end{array}$ & $\begin{array}{c}-0.011 * * * \\
(0.001)\end{array}$ \\
\hline Residente urbano & & & $\begin{array}{c}-0.109 * * * \\
(0.035) \\
\end{array}$ & $\begin{array}{c}-0.099 * * * \\
(0.035)\end{array}$ \\
\hline Democracia - Polity2 & & & & $\begin{array}{l}-0.002 \\
(0.050)\end{array}$ \\
\hline PBI per cápita & & & & $\begin{array}{c}0.000 \\
(0.000)\end{array}$ \\
\hline
\end{tabular}


Tabla 2 (cont.)

Preocupación sobre el cambio climático, situación económica y priorización del crecimiento económico en América Latina 2016

\begin{tabular}{|l|c|c|c|c|}
\hline & Modelo 1 & Modelo 2 & Modelo 3 & Modelo 4 \\
\hline Recursos Naturales/PBI & & & $\begin{array}{c}0.026 \\
(0.022)\end{array}$ \\
\hline Constante & $\begin{array}{c}2.842 * * * \\
(0.111)\end{array}$ & $\begin{array}{c}3.896 * * * \\
(0.229)\end{array}$ & $\begin{array}{c}4.855^{* * *} \\
(0.256)\end{array}$ & $\begin{array}{c}4.692 * * * \\
(0.421)\end{array}$ \\
\hline InSigma & $\begin{array}{c}-1.277 * * * \\
(0.174)\end{array}$ & $\begin{array}{c}-1.275^{* * *} \\
(0.174)\end{array}$ & $\begin{array}{c}-1.413 * * * \\
(0.177)\end{array}$ & $\begin{array}{c}-1.532 * * * \\
(0.185)\end{array}$ \\
\hline Insig_e:_cons & $\begin{array}{c}0.778^{* * *} \\
(0.004)\end{array}$ & $\begin{array}{c}0.777 * * * \\
(0.004)\end{array}$ & $\begin{array}{c}0.742 * * * \\
(0.005)\end{array}$ & $\begin{array}{c}0.740 * * * \\
(0.005)\end{array}$ \\
\hline Observaciones nivel 1 (Individuos) & 26423 & 26423 & 22819 & 21553 \\
\hline Observaciones nivel 2 (País) & 18 & 18 & 17 & 17 \\
\hline
\end{tabular}

Fuente: Elaboración propia con datos de Lapop 2016.

Nota: Modelos jerárquicos lineales. Errores estándar entre paréntesis. $* * * p<0.01, * * p<0.05, * p<0.1$

Todos los modelos incluyen las ponderaciones estándar a nivel individual del Barómetro de las Américas.

Para presentar gráficamente los principales resultados, uso las estimaciones del modelo 4, que incluye todos los controles. La Figura 2 representa el efecto de los distintos niveles de Preocupación por las consecuencias del cambio climático sobre la priorización del medioambiente para diferentes valores del índice de Riqueza patrimonial, que tiene un rango de valores entre 1 y 12, con una media de 7.228. Entre individuos más pobres con un valor inferior a 5 en el índice de riqueza patrimonial, el medioambiente no es una prioridad y los distintos niveles de preocupación por el cambio climático tienen efectos indistinguibles sobre la priorización del medioambiente. En línea con la Hipótesis 1, la preocupación por el cambio climático se asocia a priorización del medioambiente cuando los encuestados tienen una situación económica más favorable, es decir, cuando el índice de riqueza supera la media. Mientras los ciudadanos no alcanzan cierto nivel de patrimonio o de bienestar material, la variación en el nivel de preocupación por el cambio climático no tiene efectos significativos sobre las preferencias políticas con respecto a priorizar el medioambiente o el crecimiento. Modelar explícitamente la posibilidad de que la preocupación por las consecuencias del cambio climático tenga efectos diferentes sobre las preferencias con respecto a la priorización del medioambiente o el crecimiento dependiendo de la situación económica de los encuestados nos permite identificar las condiciones bajo las cuales la preocupación por el calentamiento global tiene un efecto positivo o uno negativo sobre la priorización del medioambiente. 
ALEJANDRA ARMESTO

Figura 2

Efectos marginales para Priorización del medioambiente (vs. Crecimiento económico) de la Preocupación por el Cambio Climático dependiendo de la Riqueza patrimonial

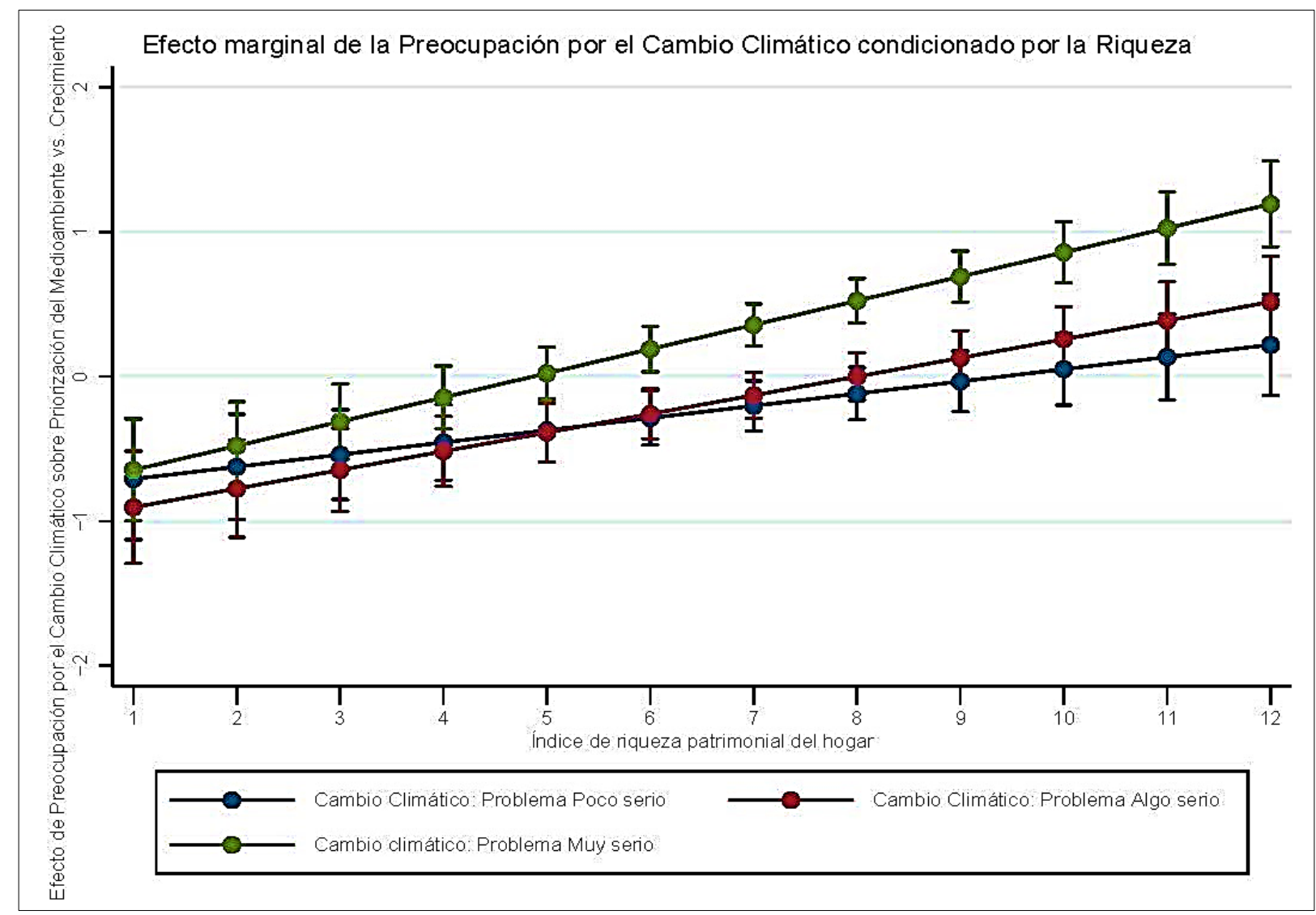

Fuente: Elaboración propia con datos de Lapop 2016.

Por último, considerando la segunda hipótesis, la Figura 2 también refleja que el efecto positivo de la riqueza sobre la priorización del medioambiente depende del nivel de preocupación por el cambio climático. Por ejemplo, entre quienes tienen el valor máximo de riqueza patrimonial, cuando las consecuencias del cambio climático se consideran serias, la priorización del medioambiente crece un punto con respecto a cuando se considera que las consecuencias no son nada serias. En suma, la riqueza patrimonial tiene un efecto positivo y estadísticamente significativo sobre la priorización del medioambiente, y este efecto es significativamente mayor para quienes consideran que el cambio climático tendrá consecuencias serias.

La Tabla 3 reporta los efectos marginales obtenidos de los modelos de ecuaciones estructurales para variable dependiente discreta con tres categorías, considerando la estructura anidada de los datos. Estos modelos incluyen las mismas variables y términos 
de interacción que los modelos jerárquicos lineales reportados en la Tabla 2. El modelo multinomial identifica los coeficientes de cada opción de respuesta en relación con una opción de referencia. A su vez, estos coeficientes dan lugar a un conjunto equivalente de efectos marginales o impactos de las variables independientes sobre las probabilidades de elegir cada una de las tres opciones cuando se pregunta a los encuestados si prefieren priorizar el medioambiente o el crecimiento económico o ambos. Las probabilidades de priorizar el medioambiente o el crecimiento económico no son independientes entre sí. A medida que aumenta la probabilidad de que se elija una de las opciones, la probabilidad de que se elijan las otras necesariamente disminuye, ya que la pregunta de la encuesta permite elegir solo una opción. Los resultados en la primera columna - A favor del medioambiente - son casi idénticos a los reportados en la Tabla 2. Si revisamos los valores de los coeficientes de la interacción entre riqueza y la preocupación más alta por el cambio climático, la probabilidad de priorizar el medioambiente es positiva y la de priorizar el crecimiento económico o ambos, crecimiento y ambiente, es negativa. Este efecto positivo de la riqueza sobre la relación entre la preocupación por el cambio climático y la probabilidad de priorizar el medioambiente proviene de la sustitución de la priorización del crecimiento económico y de la posición intermedia que pretende priorizar ambos, medioambiente y crecimiento, cuyos coeficientes son negativos.

Tabla 3

Preocupación por el Cambio climático, condiciones económicas y priorización del medioambiente o el desarrollo económico en América Latina 2016

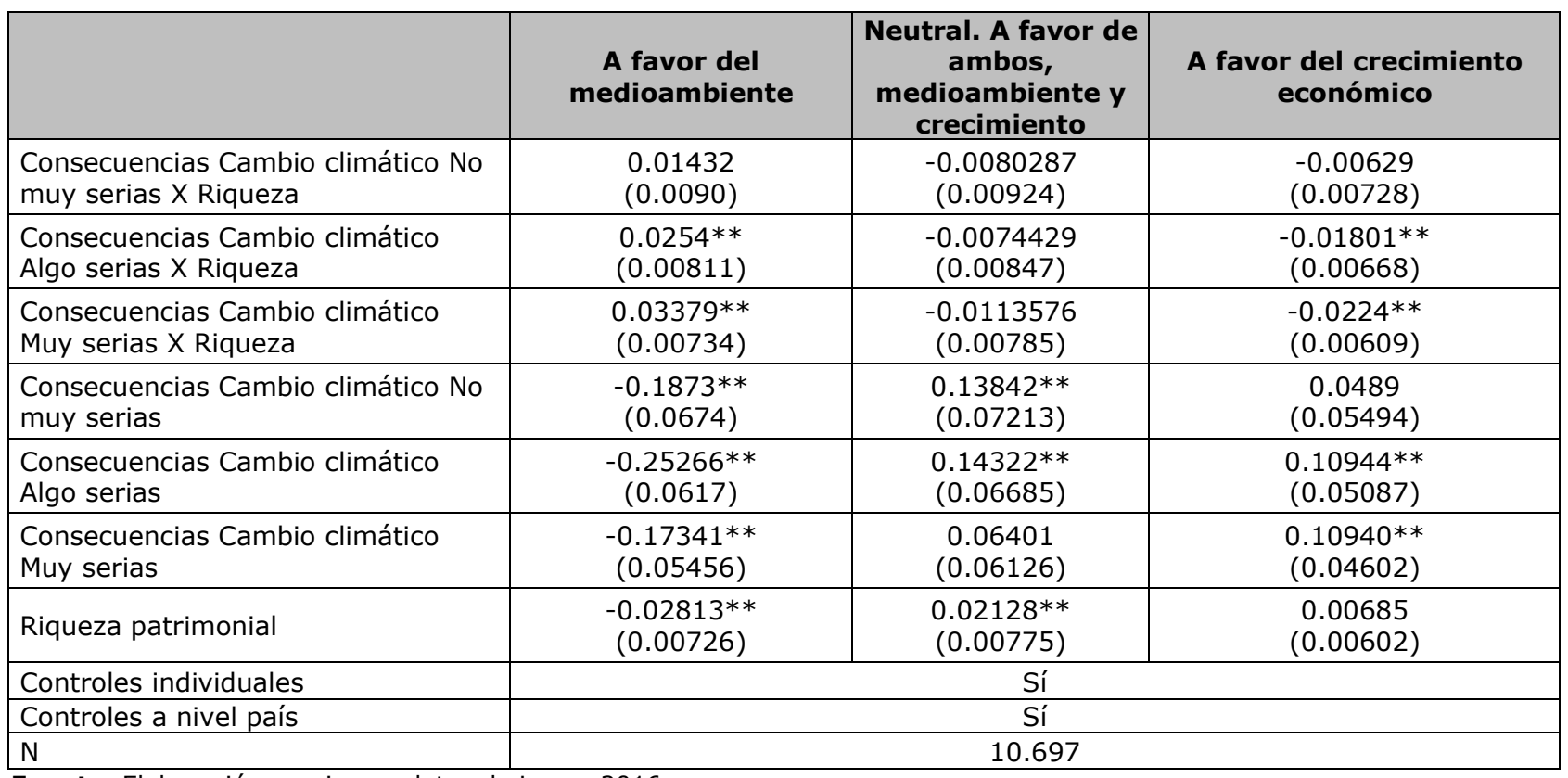

Fuente: Elaboración propia con datos de Lapop 2016.

Nota: Modelos de ecuaciones estructurales generalizadas, Multinomial. Efectos marginales dP (A favor del medioambiente/Neutral, Ambos/A favor del crecimiento económico)/dX.

Sig.: $* \mathrm{p}<.05, * * \mathrm{p}<.01, * * * \mathrm{p}<.001$. 


\section{Conclusiones}

Este artículo examinó las condiciones bajo las cuales la preocupación por el cambio climático afecta las preferencias de los ciudadanos frente al dilema entre priorizar el medioambiente o el crecimiento económico. Argumento que la percepción subjetiva de los riesgos asociados al cambio climático afecta las preferencias sobre el dilema entre medioambiente y crecimiento económico, dependiendo de la situación económica individual. Entre quienes gozan de una situación económica aventajada, la preocupación por las consecuencias serias del cambio climático tiene un efecto positivo sobre la priorización del medioambiente porque su nivel de bienestar les permite renunciar a cierto crecimiento, mientras quienes enfrentan situaciones económicas más precarias, aun cuando expresen preocupación por las eventuales consecuencias del calentamiento global, no están en condiciones de renunciar al crecimiento. En segundo lugar, el efecto de la riqueza sobre las prioridades entre medioambiente y crecimiento económico depende del nivel de preocupación por las consecuencias del cambio climático.

El artículo analiza datos de encuestas del año 2016 del proyecto Barómetro de las Américas para dieciocho países de América Latina con modelos jerárquicos lineales y ecuaciones estructurales generalizadas para variable discreta múltiple. En línea con las hipótesis propuestas, los hallazgos del estudio son los siguientes. El efecto de la preocupación por el cambio climático sobre la priorización del medioambiente depende de la situación económica. La variación en la percepción de las consecuencias del calentamiento global tiene efectos positivos sobre la priorización del medioambiente cuando las condiciones económicas individuales mejoran y se supera la media del índice de riqueza patrimonial. Asimismo, estos análisis apoyan en gran medida la hipótesis de que la preocupación por el cambio climático también modera el efecto de la riqueza en la priorización del medioambiente. Específicamente, el efecto positivo de la riqueza sobre las preferencias políticas en favor de la priorización del medioambiente se intensifica a medida que aumenta la preocupación por el cambio climático.

La investigación existente ha atendido los efectos directos de la preocupación por el cambio climático o de la situación económica sobre las actitudes y preferencias políticas ambientales. Este estudio contribuye con un argumento que pone de relieve la naturaleza condicional de los efectos de la preocupación por el cambio climático y aporta a la investigación sobre las preferencias ambientales en América Latina, donde las consecuencias del cambio climático tienen y tendrán importantes impactos sociales.

Los resultados de este estudio tienen implicaciones para futuras investigaciones comparativas sobre las causas del apoyo a políticas ambientales y los efectos actitudinales de la percepción del riesgo asociado al cambio climático, así como de factores institucionales, políticos y estructurales sobre actitudes de política ambiental. Específicamente, el hallazgo de que la preocupación por el cambio climático no impacta sobre la priorización del medioambiente en los ciudadanos menos favorecidos económicamente pone de relieve la pertinencia de investigaciones que aborden las 
PREOCUPACIÓN POR EL CAMBIO CLIMÁTICO, CONDICIONES ECONÓMICAS INDIVIDUALES Y PRIORIZACIÓN DEL MEDIOAMBIENTE..

condiciones bajo las cuales la percepción del riesgo climático moldea las actitudes y comportamientos ambientales para estos grupos especialmente vulnerables.

\section{Referencias bibliográficas}

ANDERSON, B.; Bohmelt, T.; WARD, H. "Public opinion and environmental policy output: a crossnational analysis of energy policies in Europe". Environmental Research Letters, vol. 12, no 11, p. 10, nov. 2017.

ARNOLD, J. R. "Political awareness, corruption perceptions and democratic accountability in Latin America". Acta Politica, vol. 47, no 1, p. 67-90, 2012.

ARPAD, T. "Willing to pay to save the planet? Evaluating support for increased spending on sustainable development and environmentally friendly policies in five countries". Plos One, vol. 13, no 11 , p. 15, nov. 2018.

BAEZ, J., et al. "Droughts augment youth migration in Northern Latin America and the Caribbean". Climatic Change, vol. 140, no 3-4, p. 423-435, fev. 2017.

BAKAKI, Z.; BERNAUER, T. "Do economic conditions affect public support for environmental policy?". Journal of Cleaner Production, vol. 195, p. 66-78, set. 2018.

BALDWIN, K.; HUBER, J. D. "Economic versus cultural differences: forms of ethnic diversity and public goods provision". American Political Science Review, vol. 104, no 4, p. 644-662, 2010.

BASto-ABreu, A. C., et al. "Behaviours and opinions towards outdoor smoking bans and cigarette littering in Baja California, Mexico". Health Policy and Planning, vol. 31, no 3, p. 309-313, abr. 2016.

BORISOVA, E., et al. "Social capital and preferences for redistribution to target groups". European Journal of Political Economy, vol. 54, no SI, p. 56-67, 2018.

Bou-HABIB, P. "Climate justice and historical responsibility". Journal of Politics, vol. 81, no 4, p. 1.298-1.310, out. 2019.

BRECHIN, S. R. "Objective problems, subjective values, and global environmentalism: evaluating the postmaterialist argument and challenging a new explanation". Social Science Quarterly, vol. 80, no 4, p. 793-809, dez. 1999.

BRouWER, R.; BRANDER, L.; VAN BEUKERING, P. "'A convenient truth': air travel passengers' willingness to pay to offset their CO $^{2}$ emissions". Climatic Change, vol. 90, no 3, p. 299-313, 1 out. 2008.

BURSTEIN, P. "The impact of public opinion on public policy: a review and an agenda". Political Research Quarterly, vol. 56, no 1, p. 29-40, mar. 2003.

CAO, X.; WARD, H. "Winning coalition size, state capacity, and time horizons: an application of modified selectorate theory to environmental public goods provision". International Studies Quarterly, vol. 59, no 2, p. 264-279, jun. 2015.

CHANKRAJANG, T.; MUtTARAK, R. "Green returns to education: does schooling contribute to proenvironmental behaviours? Evidence from Thailand". Ecological Economics, vol. 131, p. 434-448, jan. 2017.

DARIER, E.; SCHÜLE, R. "'Think globally, act locally'? Climate change and public participation in Manchester and Frankfurt". Local Environment, vol. 4, no 3, p. 317-329, 1999.

DAYRELL, C. "Discourses around climate change in Brazilian newspapers: 2003-2013". Discourse \& Communication, vol. 13, n² 2, p. 149-171, abr. 2019. 
DAYRELL, C.; URRY, J. "Mediating climate politics: the surprising case of Brazil". European Journal of Social Theory, vol. 18, no 3, p. 257-273, ago. 2015.

Debono, R.; VinCENTI, K.; CALlejA, N. "Risk communication: climate change as a human-health threat, a survey of public perceptions in Malta". European Journal of Public Health, vol. 22, no 1, p. 144-149, 2010.

Diekmann, A.; FRANZEN, A. "The wealth of nations and environmental concern". Environment and Behavior, vol. 31, no 4, p. 540-549, jul. 1999.

DindA, S. "Environmental Kuznets curve hypothesis: a survey". Ecological Economics, vol. 49, no 4, p. 431-455, 2004.

DREWS, S.; VAN DEN BERGH, J. C. J. M. "What explains public support for climate policies? A review of empirical and experimental studies". Climate Policy, vol. 16, nº 7, p. 855-876, 2016.

DUNLAP, R. E.; MCCRIGHT, A. M. "A widening gap: Republican and Democratic views on climate change". Environment: Science and Policy for Sustainable Development, vol. 50, nº 5, p. 26-35, 2008.

DunlaP, R. E.; MerTiG, A. G. "Global concern for the environment: is affluence a prerequisite?". Journal of Social Issues, vol. 51, no 4, p. 121-137, 1995.

. "Global environmental concern: an anomaly for postmaterialism". Social Science

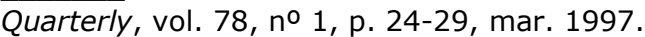

DUNLAP, R. E.; YORK, R. "The globalization of environmental concern and the limits of the postmaterialist values explanation: evidence from four multinational surveys". Sociological Quarterly, vol. 49, no 3, p. 529-563, 2008.

ELIAS, T., et al. "Media use, cross-national samples, and the theory of planned behavior: implications for climate change advocacy intentions". International Journal of Communication, vol. 13, p. 3.694$3.718,2019$.

Feng, S. Z.; KRUeger, A. B.; Oppenheimer, M. "Linkages among climate change, crop yields and Mexico-US cross-border migration". Proceedings of the National Academy of Sciences of the United States of America, vol. 107, no 32, p. 14.257-14.262, ago. 2010.

FiCKO, A.; BONCINA, A. "Public attitudes toward environmental protection in the most developed countries: the environmental concern Kuznets curve theory". Journal of Environmental Management, vol. 231, p. 968-981, fev. 2019.

FRANZEN, A. "Environmental attitudes in international comparison: an analysis of the ISSP surveys 1993 and 2000". Social Science Quarterly, vol. 84, no 2, p. 297-308, jun. 2003.

FRANZEN, A.; MeYER, R. "Environmental attitudes in cross-national perspective: a multilevel analysis of the ISSP 1993 and 2000". European Sociological Review, vol. 26, no 2, p. 219-234, 2009.

FRANZEN, A.; VoGL, D. "Acquiescence and the willingness to pay for environmental protection: a comparison of the ISSP, WVS, and EVS". Social Science Quarterly, vol. 94, n 3, p. 637-659, set. 2012.

" "Two decades of measuring environmental attitudes: a comparative analysis of 33 countries". Global Environmental Change, vol. 23, no 5, p. 1.001-1.008, 2013.

GELISSEN, J. "Explaining popular support for environmental protection: a multilevel analysis of 50 nations". Environment and Behavior, vol. 39, no 3, p. 392-415, 2007. 
PREOCUPACIÓN POR EL CAMBIO CLIMÁTICO, CONDICIONES ECONÓMICAS INDIVIDUALES Y PRIORIZACIÓN DEL MEDIOAMBIENTE...

Graham, H., et al. "Willingness to pay for policies to reduce future deaths from climate change: evidence from a British survey". Public Health, vol. 174, p. 110-117, 2019.

GUBER, D. L. Partisan cueing and polarization in public opinion about climate change. In: NISBET, M. et al. The Oxford Encyclopedia of Climate Change Communication. Oxford: Oxford University Press, 2017.

HAMILTON, L., et al. "Tracking public beliefs about anthropogenic climate change". PloS One, vol. 10, p. e0138208, 2015.

HAPPER, C.; Wellesley, L. "Meat consumption, behaviour and the media environment: a focus group analysis across four countries". Food Security, vol. 11, no 1, p. 123-139, fev. 2019.

HARRING, N.; JAGERS, S. C.; MATTI, S. "Public support for pro-environmental policy measures: examining the impact of personal values and ideology". Sustainability, vol. 9, n 5, p. 14, maio 2017.

HERSCH, J.; VISCUSI, W. K. "The generational divide in support for environmental policies: European evidence". Climatic Change, vol. 77, no 1-2, p. 121-136, jul. 2006.

HINCHLIFFE, S. "Helping the earth begins at home: the social construction of socio-environmental responsibilities". Global Environmental Change, vol. 6, no 1, p. 53-62, 1996.

INGLEHART, R. The silent revolution: changing values and political styles among Western publics. Princeton, NJ: Princeton University Press, 1977.

. "Post-materialism in an environment of insecurity". American Political Science Review, vol.

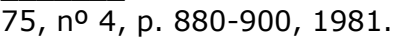

. "Aggregate stability and individual-level flux in mass belief systems: the level of analysis paradox". American Political Science Review, vol. 79, no 1, p. 97-116, 1985.

"Public support for environmental protection: objective problems and subjective values in 43 societies". PS: Political Science \& Politics, vol. 28, no 1, p. 57-72, 1995.

Inglehart, R.; ABramson, P. R. "Economic security and value change". American Political Science Review, vol. 88, no 2, p. 336-354, 1994.

JOHNSON, E. W.; SCHWADEL, P. "It is not a cohort thing: interrogating the relationship between age, cohort, and support for the environment". Environment and Behavior, vol. 51, no 7, p. 879-901, ago. 2019.

Kachi, A.; Bernauer, T.; Gampfer, R. "Climate policy in hard times: are the pessimists right?". Ecological Economics, vol. 114, p. 227-241, jun. 2015.

Kellstedt, P.; Zahran, S.; Vedlitz, A. "Personal efficacy, the information environment, and attitudes toward global warming and climate change in the United States". Risk Analysis: An official publication of the Society for Risk Analysis, vol. 28, p. 113-126, 2008.

KöLLN, A.-K. "Political sophistication affects how citizens' social policy preferences respond to the economy". West European Politics, vol. 41, no 1, p. 196-217, 2018.

KUZNETS, S. "Economic growth and income inequality". The American Economic Review, vol. 45, no 1 , p. 1-28, 1955.

Kvaloy, B.; FinseraAs, H.; Listhaug, O. "The publics' concern for global warming: a cross-national study of 47 countries". Journal of Peace Research, vol. 49, no 1, p. 11-22, jan. 2012. 
LEISEROWITZ, A. "Climate change risk perception and policy preferences: the role of affect, imagery, and values". Climatic Change, vol. 77, no 1-2, p. 45-72, jul. 2006.

LERGETPORER, P., et al. "How information affects support for education spending: evidence from survey experiments in Germany and the United States". Journal of Public Economics, vol. 167, p. 138-157, 2018.

LoRenzoni, I.; NiCHOLSON-COLE, S.; WhitMARSH, L. "Barriers perceived to engaging with climate change among the UK public and their policy implications". Global Environmental Change, vol. 17, n० 3, p. 445-459, 2007.

LUBELL, M., et al. "Collective action, environmental activism, and air quality policy". Political Research Quarterly, vol. 59, no 1, p. 149-160, mar. 2006.

MALKA, A.; LeLKES, Y. "More than ideology: conservative-liberal identity and receptivity to political cues". Social Justice Research, vol. 23, n 2-3, p. 156-188, 2010.

MAYER, A.; SMITH, E. K. "Unstoppable climate change? The influence of fatalistic beliefs about climate change on behavioural change and willingness to pay cross-nationally". Climate Policy, vol. 19, n० 4, p. 511-523, abr. 2019.

MCCRIGHT, A. M.; DUNLAP, R. E. "Bringing ideology in: the conservative white male effect on worry about environmental problems in the USA". Journal of Risk Research, vol. 16, n० 2, p. 211-226, 2013.

MCCRIGHT, A. M.; XIAO, C. "Gender and environmental concern: insights from recent work and for future research". Society \& Natural Resources, vol. 27, n 10, p. 1.109-1.113, 2014.

Mildenberger, M.; Tingley, D. "Beliefs about climate beliefs: the importance of second-order opinions for climate politics". British Journal of Political Science, vol. 49, no 4, p. 1.279-1.307, out. 2019.

MitTIGA, R. Allocating the burdens of climate action: consumption-based carbon accounting and the polluter-pays principle. In: EDMONDSON, B.; LEVY, S. (eds.). Transformative climates and accountable governance (Palgrave Studies in Environmental Transformation Transition and Accountability). Basingstoke: Palgrave, p. 157-194, 2019.

NeUmAYER, E. "In defence of historical accountability for greenhouse gas emissions". Ecological Economics, vol. 33, no 2, p. 185-192, 2000.

. "The environment, left-wing political orientation and ecological economics". Ecological Economics, vol. 51, no 3-4, p. 167-175, 2004.

NiSBET, M. C. "Communicating climate change: why frames matter for public engagement". Environment: Science and Policy for Sustainable Development, vol. 51, no 2, p. 12-23, 2009.

O'CONNOR, R. E.; BORD, R. J.; FISHER, A. "Risk perceptions, general environmental beliefs, and willingness to address climate change". Risk Analysis, vol. 19, no 3, p. 461-471, jun. 1999.

O'CONNOR, R. E., et al. "Who wants to reduce greenhouse gas emissions?". Social Science Quarterly, vol. 83, no $^{\circ}$, p. 1-17, 2002.

OSTMAN, R. E.; PARKER, J. L. "Impact of education, age, newspapers, and television on environmental knowledge, concerns, and behaviors". The Journal of Environmental Education, vol. 19, no 1, p. 3-9, 1987.

PAGE, B.; ShAPIRO, R. "Effects of public opinion on policy". The American Political Science Review, vol. 77, no 1, p. 175-190, 1983. 
PREOCUPACIÓN POR EL CAMBIO CLIMÁTICO, CONDICIONES ECONÓMICAS INDIVIDUALES Y PRIORIZACIÓN DEL MEDIOAMBIENTE...

Post, D.; MENG, Y. "Does schooling foster environmental values and action? A cross-national study of priorities and behaviors". International Journal of Educational Development, vol. 60, p. 10-18, maio 2018.

PovitkinA, M. "The limits of democracy in tackling climate change". Environmental Politics, vol. 27, no 3, p. 411-432, 2018.

PugAtCH, T. "Tropical storms and mortality under climate change". World Development, vol. 117, p. 172-182, maio 2019.

Raudenbush, S. W.; Bryk, A. S. Hierarchical linear models. 2 a ed. Newbury Park, CA: Sage, 2002.

RuBin, O.; Rossing, T. "National and local vulnerability to climate-related disasters in Latin America: the role of social asset-based adaptation". Bulletin of Latin American Research, vol. 31, no 1, p. 1935, jan. 2012.

SLOVIC, P. "Perception of risk". Science, vol. 236, n 4.799, p. 280-285, 1987.

SNIJDERS, T. A. B.; BOSKER, R. J. Multilevel analysis: an introduction to basic and advanced multilevel modeling. London: Sage, 1999.

Sonnenschein, J.; SMEdBy, N. "Designing air ticket taxes for climate change mitigation: insights from a Swedish valuation study". Climate Policy, vol. 19, no 5, p. 651-663, maio 2019.

StenNer, K.; NWOKorA, Z. "Current and future friends of the earth: assessing cross-national theories of environmental attitudes". Energies, vol. 8, no 6, p. 4.899-4.919, jun. 2015.

STOKeS, B.; WIKE, R.; CARLE, J. Global concern about climate change, broad support for limiting emissions. Washington, D.C.: Pew Research Center, nov. 2015.

StREIMIKIENE, D., et al. "A review of willingness to pay studies for climate change mitigation in the energy sector". Energies, vol. 12, no 8, p. 38, abr. 2019.

TJernstrom, E.; Tietenberg, T. "Do differences in attitudes explain differences in national climate change policies?". Ecological Economics, vol. 65, no 2, p. 315-324, abr. 2008.

VISCUSI, W. K.; ZECKHAUSER, R. J. "The perception and valuation of the risks of climate change: a rational and behavioral blend". Climatic Change, vol. 77, no 1-2, p. 151-177, jul. 2006.

WARD, H. "Liberal democracy and sustainability". Environmental Politics, vol. 17, no 3, p. 386-409, jun. 2008.

WURSTER, S. "Comparing ecological sustainability in autocracies and democracies". Contemporary Politics, 19, no 1, p. 76-93, mar. 2013.

ZAHRAN, S., et al. "Climate change vulnerability and policy support". Society \& Natural Resources, vol. 19, no 9, p. 771-789, 2006.

\section{Anexo metodológico}

Datos

Utilizo las encuestas del Barómetro de las Américas (Lapop) de 18 países latinoamericanos administradas en 2016 porque los cuestionarios de este año incluyen preguntas sobre la preocupación 
con respecto al cambio climático y la priorización del medioambiente o el crecimiento para todos los países. Los datos para este documento consisten en 26.423 respuestas de encuestas realizadas en los siguientes países: Argentina, Bolivia, Brasil, Chile, Colombia, Costa Rica, República Dominicana, Ecuador, El Salvador, Guatemala, Honduras, México, Nicaragua, Panamá, Paraguay, Perú, Uruguay y Venezuela.

\section{Variable dependiente}

Preferencias frente a la disyuntiva entre priorizar el medioambiente o el crecimiento económico: pregunta de Lapop ENV1. Alguna gente cree que hay que priorizar la protección del medioambiente sobre el crecimiento económico, mientras otros creen que el crecimiento económico debería priorizarse sobre la protección ambiental. En una escala de 1 a 7 en la que 1 significa que el medioambiente debe ser la principal prioridad, y 7 significa que el crecimiento económico debe ser la principal prioridad, ¿dónde se ubicaría usted? Para facilitar la interpretación de la preferencia de priorizar el medioambiente he invertido la escala, de modo que 1 refleja la priorización del crecimiento económico y 7 la priorización del medioambiente.

\section{Variables independientes}

Preocupación por el cambio climático: pregunta de Lapop ENV2B1. Si no se hace nada para reducir el cambio climático en el futuro, ¿qué tan serio piensa usted que sería el problema para (País)? Las opciones de respuesta son: (1) Muy serio, (2) Algo serio, (3) Poco serio y (4) Nada serio. Para esta variable también he invertido la escala para facilitar la interpretación.

Situación económica individual: índice de riqueza resultante de un análisis de componentes principales con respuestas a preguntas sobre la presencia de los siguientes elementos: televisión, refrigerador, teléfono convencional, teléfono celular, vehículo/automóvil, lavadora, horno de microondas, motocicleta, agua potable, baño dentro de la casa y computadora (preguntas de Lapop R1, R3, R4, $R 4 A, R 6, R 7, R 8, R 12, R 14, R 15, R 18)$.

Variables de control

Evaluación de la situación económica personal: pregunta de Lapop IDIO2. Considera usted que su situación económica actual es ¿mejor, igual o peor que la de hace doce meses? (1) Mejor (2) Igual (3) Peor.

Evaluación de la situación económica nacional: pregunta de Lapop SOCT2. ¿Considera usted que la situación económica del país es mejor, igual o peor que hace doce meses? (1) Mejor (2) Igual (3) Peor. 
PREOCUPACIÓN POR EL CAMBIO CLIMÁTICO, CONDICIONES ECONÓMICAS INDIVIDUALES Y PRIORIZACIÓN DEL MEDIOAMBIENTE...

Eficacia política: pregunta de Lapop EFF1. A los que gobiernan el país les interesa lo que piensa la gente como usted. ¿Hasta qué punto está de acuerdo o en desacuerdo con esta frase? Entre 1 (Muy en desacuerdo) y 7 (Muy de acuerdo).

Eficacia personal: pregunta de Lapop EFF2. Usted siente que entiende bien los asuntos políticos más importantes del país. ¿Hasta qué punto está de acuerdo o en desacuerdo con esta frase? Entre 1 (Muy en desacuerdo) y 7 (Muy de acuerdo).

Ideología: pregunta de Lapop L1. Cambiando de tema, en esta tarjeta tenemos una escala del 1 a 10 que va de izquierda a derecha, en la que el 1 significa izquierda y el 10 significa derecha. Hoy en día cuando se habla de tendencias políticas, mucha gente habla de aquellos que simpatizan más con la izquierda o con la derecha. Según el sentido que tengan para usted los términos "izquierda" y "derecha" cuando piensa sobre su punto de vista político, ¿dónde se encontraría usted en esta escala?.

Exposición a medios de comunicación: pregunta de Lapop GI0. ¿Con qué frecuencia sigue las noticias, ya sea en la televisión, la radio, los periódicos o el Internet? (1) Diariamente (2) Algunas veces a la semana (3) Algunas veces al mes (4) Rara vez (5) Nunca.

Presencia de niños en el hogar: pregunta de Lapop Q12BN. ¿Cuántos niños menores de 13 años viven en este hogar? Recodificada en 0 y 1 ( 1 o más).

Democracia: índice Polity2

Nivel de desarrollo económico: PIB per cápita

Dependencia de los recursos naturales: suma de las rentas del petróleo, el gas natural, el carbón, los minerales y bosques como proporción del PIB.

\begin{abstract}
Concern about climate change, individual economic conditions and environment prioritization in Latin America

Latin American citizens are more concerned about the consequences of climate change than citizens of any other region in the world. However, this concern does not always lead to prioritizing the environment over economic growth. This paper argues that individuals' economic constraints moderate the relationship between their beliefs about the seriousness of climate change and their preferences with respect to the dilemma between prioritizing the environment or economic growth. The analyses of 2016 Americas Barometer (Lapop) surveys in 18 Latin American countries, with hierarchical linear models and generalized structural equations, show that the level of concern about climate change has a significant positive effect on the prioritization of the environment among individuals with above-average wealth and a negative effect among individuals with below-average wealth. This work makes two contributions. First, the study analyzes the conditional effect of concern about climate change on environment prioritization depending on individual economic constraints; and secondly, it contributes to scholarship about environmental attitudes in Latin America.
\end{abstract}


Keywords: environmental attitudes; concern about climate change; environment prioritization; economic constraints; hierarchical linear models; Latin America

\section{Resumo}

Preocupação com as mudanças climáticas, condições econômicas individuais e priorização do meio ambiente na América Latina

Os cidadãos da América Latina estão mais preocupados com as consequências das mudanças climáticas do que os de qualquer outra região do mundo. No entanto, essa preocupação nem sempre leva a priorizar o meio ambiente sobre o crescimento econômico. Este artigo argumenta que as restrições econômicas dos indivíduos condicionam a relação entre suas crenças sobre a gravidade das consequências das mudanças climáticas e suas preferências a respeito do dilema entre priorizar o meio ambiente ou o crescimento econômico. A análise dos levantamentos de 2016 do Barômetro das Américas (Lapop) em 18 países latino-americanos, com modelos hierárquicos lineares e equações estruturais generalizadas, mostra que a preocupação com as mudanças climáticas tem um efeito positivo significativo sobre a priorização do meio ambiente entre indivíduos com riqueza acima da média e um efeito negativo entre indivíduos com riqueza abaixo da média. Este artigo traz duas contribuições. Em primeiro lugar, o estudo analisa o efeito condicionado da preocupação pela mudança climática sobre a priorização do meio ambiente dependendo da situação econômica individual e, em segundo lugar, contribui para o conhecimento das atitudes ambientais na América Latina.

Palavras-chave: atitudes ambientais; preocupação com as mudanças climáticas; priorização ambiental; restrições econômicas; modelos lineares hierárquicos; América Latina

\section{Résumé}

La préoccupation pour le changement climatique, les conditions économiques individuelles et la priorisation environnementale en Amérique Latine

Les citoyens latino-américains sont plus préoccupés par les conséquences du changement climatique que ceux de toute autre région du monde. Cependant, cette préoccupation ne conduit pas toujours à privilégier l'environnement par rapport à la croissance économique. Cette article fait valoir que les contraintes économiques des individus conditionnent la relation entre leurs croyances sur la gravité du changement climatique et leurs préférences face au dilemme entre la priorité à l'environnement ou la croissance économique. Les analyses des enquêtes Lapop du Baromètre des Amériques 2016 dans 18 pays d'Amérique latine, avec des modèles linéaires hiérarchiques et des équations structurelles généralisées, montrent que le niveau d'inquiétude concernant le changement climatique a un effet positif et significatif sur la priorisation de l'environnement chez les individus avec des richesse, et un effet negative chez les individus dont la richesse est inférieure à la moyenne. Ce travail apporte deux contributions: l'étude analyse l'effet conditionnel de la préoccupation pour le changement climatique sur la priorisation de l'environnement en fonction des contraintes économiques individuelles; et deuxièmement, il contribue à la recherche sur les attitudes environnementales en Amérique latine.

Mots-clés: attitudes environnementales; préoccupation pour le changement climatique; priorité de l'environnement; contraintes économiques; modèles linéaires hiérarchiques; Amérique Latine

Artigo submetido à publicação em 19 de novembro de 2019. Versão final aprovada em 7 de janeiro de 2021.

Opinião Pública adota a licença Creative Commons CC-BY.

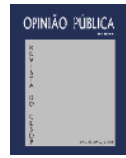

